\title{
APPENDIX 5
}

FAGAN'S NOMOGRAPH FOR APPROXIMATE EVALUATION OF POST-TEST PROBABILITIES OF DM1 WITH KNOWN LIKELIHOOD COEFFICIENTS OF ANTIBODY TESTS [13]

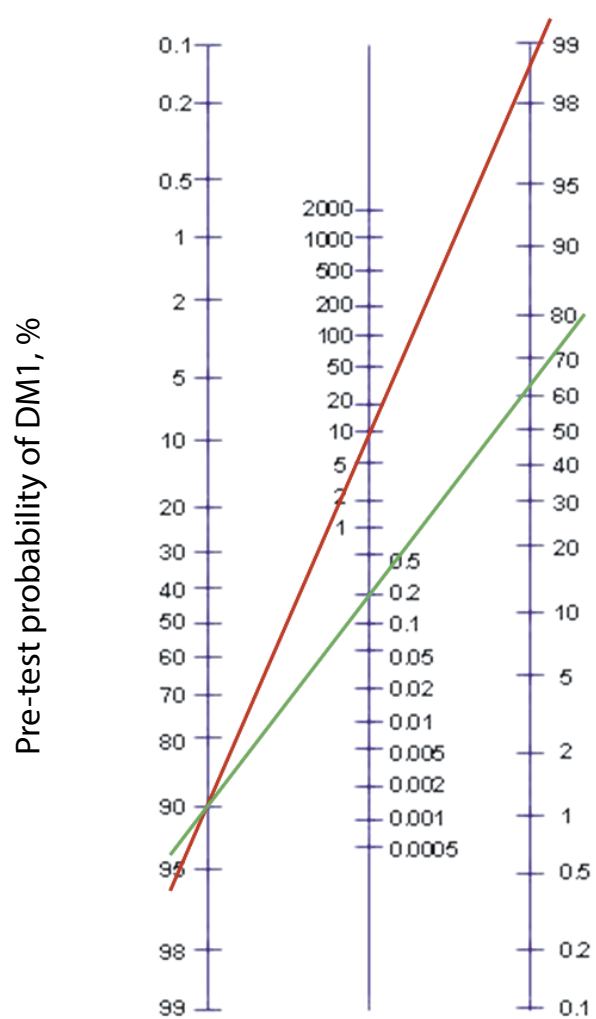

Likelihood coefficient
The left axis of the nomograph is PrP of DM1; the middle axis is the likelihood coefficient (LC) of the antibody test; and the right axis is PtP of DM1.

To determine the PtP of DM1, it is necessary to evaluate its PrP (based on literature data, experience and intuition) and to know the LC of a specific test result. On the left axis, the point corresponding to PrP of DM1 is chosen, and on the middle axis the point corresponding to the $\mathrm{LC}$ is chosen. Through these two points, a straight line is drawn. The point of intersection of the straight line with the right axis corresponds to the PtP of DM1.

The figure illustrates an example of the assessment of PtP of DM1 at its PrP of $90 \%$. We used an antibody test with $L C+=10$ and $L C-=0.2$. If the positive result of the test of PtP of DM1 is approximately $99 \%$, with a negative result, then PtP of DM1 is approximately $64 \%$. 